\section{Evidence-based guidelines improve testicular-cancer management}

EBM

Evidence-based, consensus guidelines for managing testicular cancer were implemented across Europe in 1999. The influence of these guidelines on the management of testicular cancer has been retrospectively studied by researchers who compared a cohort of patients treated before the guidelines were implemented, with a cohort of patients treated at the same tertiary referral center in Germany after the guidelines were implemented.

The records of 318 patients with germ-cell tumors were analyzed: 234 were treated between 1990 and 1999, and 84 were treated between 2000 and 2004 . In the pre-guidelines group, $17.5 \%$ of patients were overtreated, compared with $8.3 \%$ of the post-guidelines group (four patients in the latter group were treated according to a multicenter trial protocol). Undertreatment resulted in more relapses occurring in the pre-guidelines than the post-guidelines group $(8.1 \%$ versus $3.6 \%$ ), although numbers were too low to reach statistical significance. Undertreatment should be avoided, as relapse is associated with higher toxicity and mortality rates than occur in primary cancer. In the pre-guidelines group 10.3\% of patients were undertreated, compared with none in the post-guidelines group.

Notably, implementation of the guidelines decreased the mortality rate even in a specialist center, where the mortality rate was already lower than in other institutions. To improve cancer-specific mortality rates further, say the authors, we must reduce the delay between recognition of symptoms and starting treatment-a factor which might be partly to blame for the much higher mortality rate for testicular cancer seen in Europe than in the US.

Caroline Barranco

Original article Schrader AJ et al. (2006) Impact of evidence-based interdisciplinary guidelines on testis cancer management. Cancer 106: 313-319

\section{Pediatric renal transplant recipients should be monitored for bladder dysfunction}

Children receiving renal transplants as a result of pre-existing urological disease are prone to develop lower urinary tract symptoms (LUTS) postoperatively. As bladder dysfunction can damage the new kidney, these patients must be monitored carefully. To determine whether children undergoing kidney transplantation because of nephrological disease should also be rigorously observed for such problems, researchers in The Netherlands studied LUTS in both groups.

The study included 30 of 59 children who received a renal transplant at a medical center in Nijmegen, The Netherlands, between January and May 2003. Of these 30 patients (16 male, mean age at data collection 14 years), 21 had underlying nephrological disease and 9 had underlying urological disease.

Frequency volume data were available for 23 children. Increased postoperative bladder capacity - which can lead to myogenic failure and incontinence-was seen in 12/16 children with nephrological disease and 6/7 urological disease patients. Mean bladder capacities in these children, corrected for growth retardation, were $175 \%$ and $207 \%$ of normal, respectively. LUTS occurred very frequently, with only one patient experiencing none; presence of residual urine, urinary tract infection and hesitancy were particularly common events. Despite having larger bladder capacities than their younger counterparts, patients who were older at transplant and older at data collection experienced less incontinence.

Because of the high incidence of LUTS and increased bladder capacity in both groups of patients, the authors recommend that all children receiving renal transplants should be monitored for bladder dysfunction. Larger studies are needed to define which patient characteristics are useful indicators of bladder dysfunction following renal transplantation.

Rebecca Ireland

Original article Van der Weide MJA et al. (2006) Lower urinary tract symptoms after renal transplantation in children. JUrol 175: 297-302

\section{Proteinuria detected in men following sexual intercourse might be misleading}

Sexual intercourse can cause proteinuria in men for up to $12 \mathrm{~h}$, according to a small prospective study at the Israel Naval Medical Institute. When dipstick urine tests are positive for protein, further investigations must be undertaken. To avoid 